\title{
PEMAKAIAN CHELATING MONOLITH SEBAGAI SOLID PHASE MICROEXTRACTION (SPME) TRACE ELEMEN DI DALAM AIR
}

\author{
Dwinna Rahmi \\ Depertemen Perindustrian, Badan Penelitian dan Pengembangan Industri, \\ Balai Besar Kimia dan Kemasan, Jl. Balai Kimia No. 1 Pekayon Jakarta Timur, \\ Telp: (021) 8717438, Faks: (021) 8714928 \\ E-mail : dwinna2002@yahoo.com
}

\begin{abstract}
A syringe-based sample pretreatment tool, named herein "chelating monolith", has been developed for simple and facile solid phase microextraction (SPME) of trace elements in natural waters. The monolith was directly prepared within the confines of a commercially available syringe filter tip by a two-step process: 1) in situ polymerization of glycidyl methacrylate (GMA) with ethylene glycol dimethacrylate (EDMA) and 2) subsequent modification with iminodiacetate (IDA) via ring opening reaction of epoxide. The composition of porogenic solvent was first optimized to make a rigid-porous material that has high permeability and ample surface area as much as possible. Then, the $\mathrm{pH}$ and concentration of the IDA modification solution were examined to obtain higher chelating capacity. The metal adsorption properties of the obtained chelating monolith were evaluated through an adsorption/desorption experiment. After optimization of some parameters such as sample solution $\mathrm{pH}$, eluent concentration and the volume, good recoveries of more than $80 \%$ were obtained for 28 elements including REEs, $\mathrm{Fe}, \mathrm{Co}, \mathrm{Ni}, \mathrm{Cu}, \mathrm{Zn}, \mathrm{Ga}, \mathrm{Pb}$ and $\mathrm{Th}$ in a single extraction step. The proposed SPME method was validated through the analysis of river water certified reference material (CRM: JSAC 0301-1)
\end{abstract}

Keywords: Solid phase microextraction; syringe filter; chelating monolith; iminodiacetate; ICP$M S$

\section{DAFTAR PUSTAKA}

1. M. J. Bloxham, S. J. Hill, P. J. Worsfold, J. Anal. Atom. Spectrom., 9: 935, (1994).

2. H. Sawatari, T. Hayashi, E. Fujimori, A. Hirose, H. Haraguchi, Bull. Chem. Soc. Jpn., 69: 1925, (1996).

3. T. Yabutani, K. Chiba, H. Haraguchi, Bull. Chem. Soc. Jpn., 74: 31, (2001).

4. J. L. Manzoori, F. Shemirani, J. Anal. Atom. Spectrom., 10: 881, (1995)

5. M. Camino, M. G. Bagur, M. Sanchez Vinas, D. Gazquez, R. Romero, J. Anal. Atom. Spectrom., 16: 638, (2001).

6. S. Kagaya, Z. A. Malek, Y. Araki, K. Hasegawa, Anal. Sci., 18: 923, (2002).

7. N. Tokman, S. Akman, Y. Bakircioglu, Microchim. Acta, 146: 31, (2004).

8. T. Minami, Y. Sohrin, J. Ueda, Anal. Sci., 21: 1519, (2005).

9. R. A. Nickson, S. J. Hill, P. J. Worsfold,
Anal. Chim. Acta, 351: 311, (1997).

10. Y. Guo, B. Din, Y. W. Liu, X. J. Chang, S. M. Meng, M. Z. Tian, Anal. Chim. Acta, 504: 319, (2004).

11. S. Saracoglu, L. Elci, Anal. Chim. Acta, 452: 77, (2002).

12. Y. Q. Cai, G. B. Jiang, J. F. Liu, Talanta, 57: 1173, (2002).

13. K. Oshita, S. Motomizu, Bunseki Kagaku, 57: 291, (2008).

14. W. M. Landing, C.Haraldsson, N. Paxeus, Anal. Chem., 58: 3031, (1986).

15. V. A. Lemos, P. X. Baliza, Talanta, 67: 564, (2005).

16. S. D. Çekiç, H. Filik, R. Apak, Anal. Chim. Acta, 505: 15, (2004).

17. M. L. Firdaus, K. Norisuye, T. Sato, S. Urushihara, Y. Nakagawa, S. Umetani, Y. Sohrin, Anal. Chim. Acta, 583: 296, (2007).

18. S. Hjérten, J. L. Liao, R. Zhang, J. 
Chromatogr., 473: 273, (1989).

19. F. Svec, J. M. J. Fréchet, Anal. Chem., 54: $820,(1992)$.

20. C. Ericson, S. Hjertén, Anal. Chem., 71: 1621, (1999).

21. A. K. Palm, M. V. Novotny, Rapid Commun. Mass Spectrom., 18: 1374, (2004).

22. E. F. Hilder, F. Svec, J. M. J. Fréchet, Anal. Chem., 76: 3887, (2004).
23. G. Hlözl, H. Oberacher, S. Pitsch, A. Stutz, C.G. Huber, Anal. Chem., 77: 673, (2005).

24. S. Zhang, X. Huang, J. Zhang, C. Horváth, J. Chromatogr. A, 887: 465, (2000).

25. H. Minakuchi, K. Nakanishi, N. Soga, N. Ishizuka, N. Tanaka, Anal. Chem., 68: 3498, (1996).

26. Y. Zhu, R. Hattori, E. Fujimori, T. Umemura, H. Haraguchi, Anal. Sci., 21: 199, (2005). 